\title{
Holey Cobalt-Iron Nitride Nanosheet Arrays as High-Performance Bifunctional Electrocatalysts for Overall Water Splitting
}

Di Li, Yingying Xing ${ }^{b}$, Rong Yang ${ }^{b}$, Tai Wen ${ }^{b}$, Deli Jiang ${ }^{b, *}$, Weidong Shib,, Shouqi Yuan $^{a}$

${ }^{a}$ Institute for Energy Research, Jiangsu University, Zhenjiang 212013, China

${ }^{b}$ School of Chemistry and Chemical Engineering, Jiangsu University, Zhenjiang 212013, China 


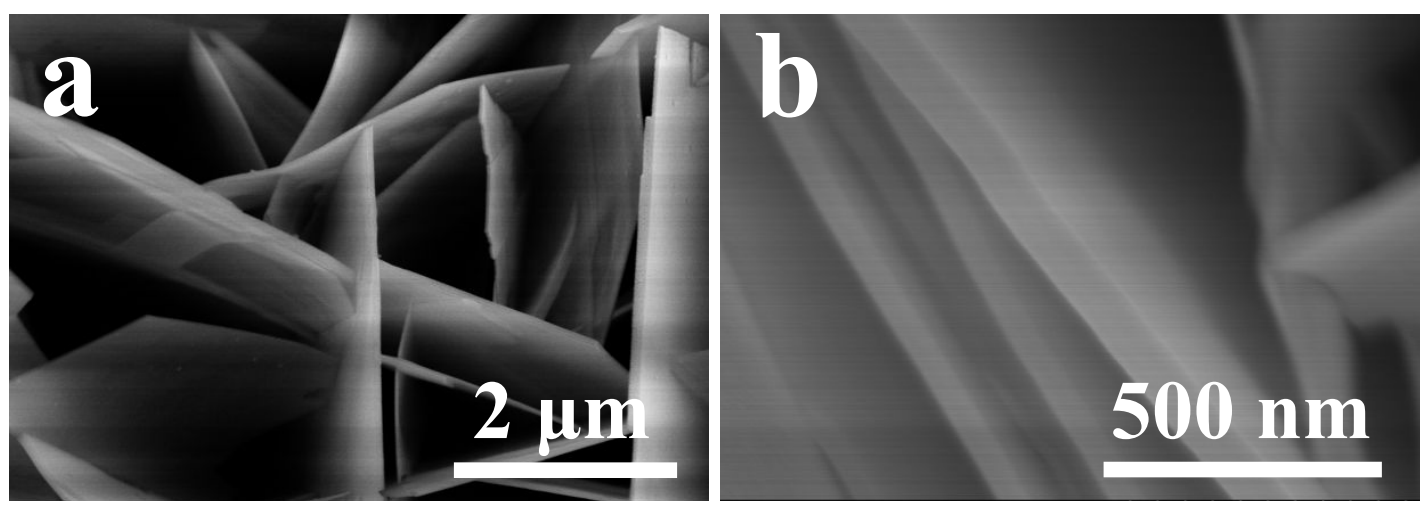

Figure S1. SEM images of $(a, b)$ CoFe-LDH/NF . 


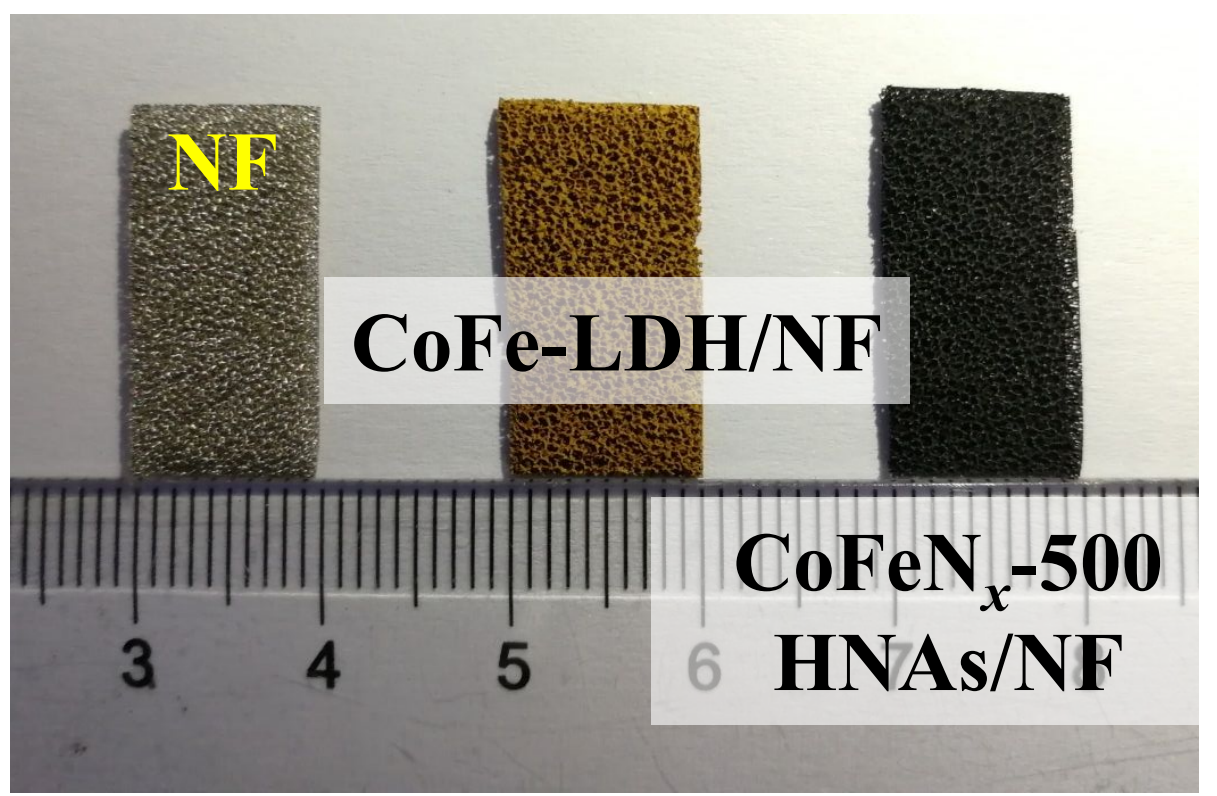

Figure S2. The photographs of bare NF, CoFe-LDH/NF and $\mathrm{CoFeN}_{x}-500 \mathrm{HNAs} / \mathrm{NF}$. 

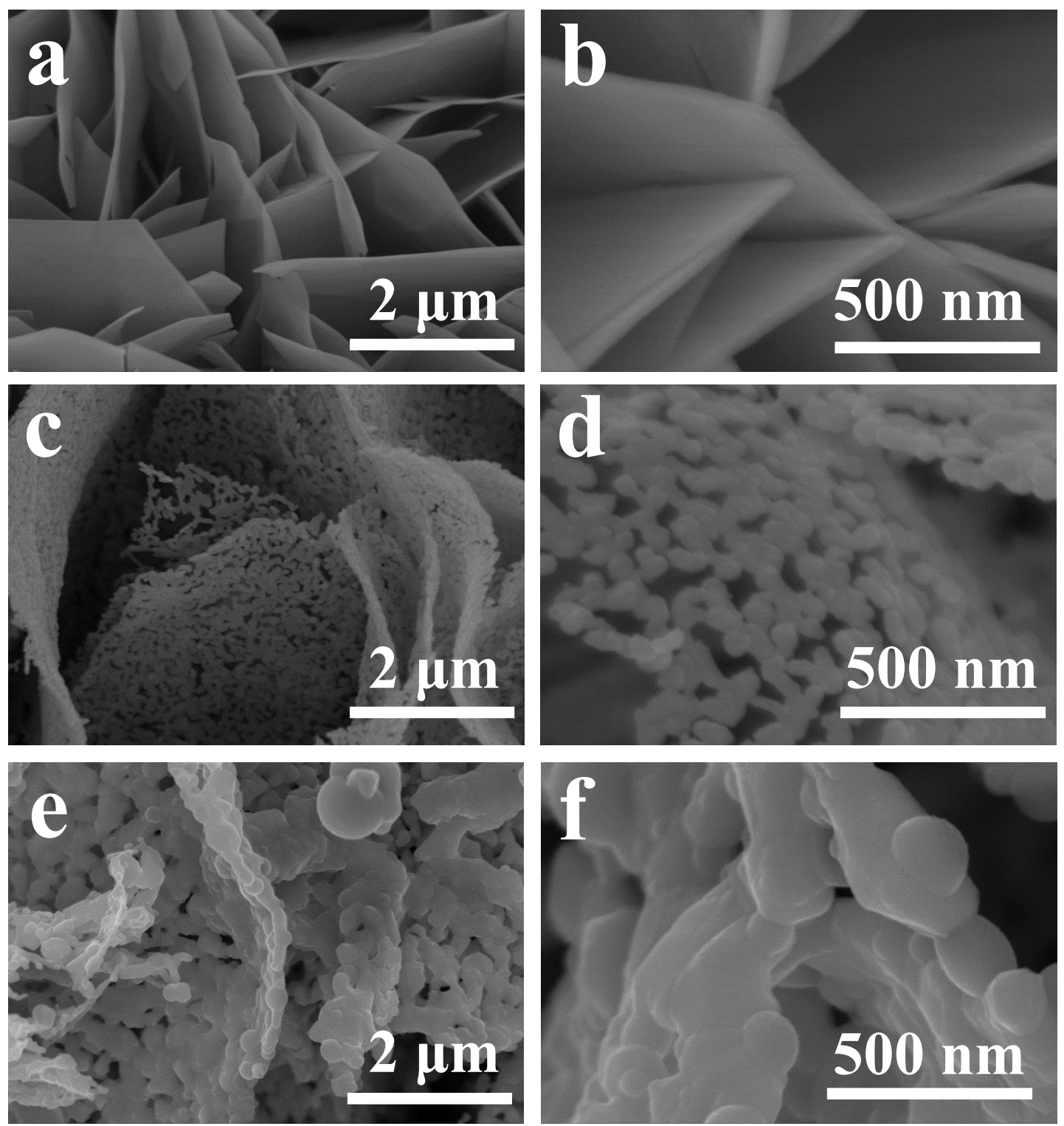

Figure S3. SEM images of (a,b) $\mathrm{CoFeN}_{x}-300 \mathrm{HNAs} / \mathrm{NF},(\mathrm{c}, \mathrm{d}) \mathrm{CoFeN}_{x}-400 \mathrm{HNAs} / \mathrm{NF},(\mathrm{e}, \mathrm{f})$ $\mathrm{CoFeN}_{x}-600$ HNAs/NF. 

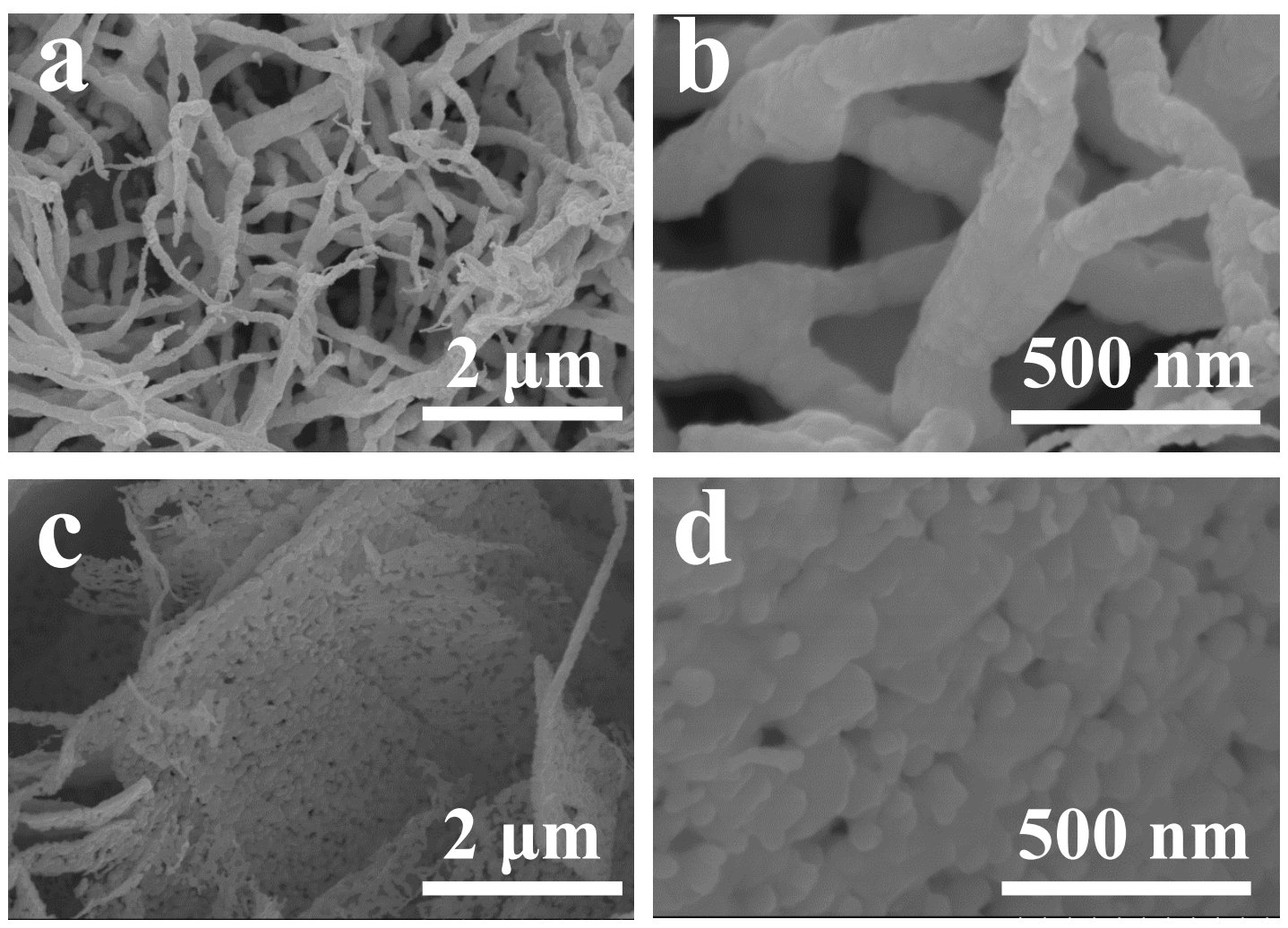

Figure S4. SEM images of $(a, b) \mathrm{Co}_{2} \mathrm{~N} / \mathrm{NF}$ and $(\mathrm{c}, \mathrm{d}) \mathrm{Fe}_{4} \mathrm{~N} / \mathrm{NF}$. 

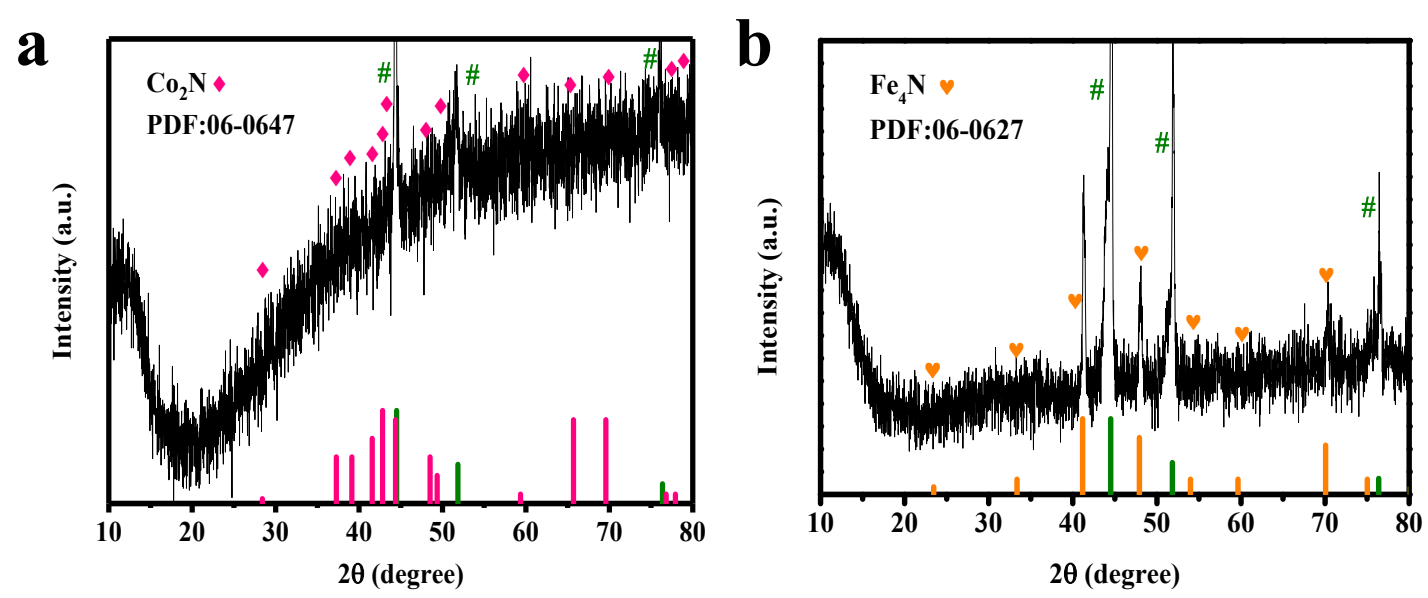

Figure S5. XRD patterns of the as-prepared samples grown on NF: (a) $\mathrm{Co}_{2} \mathrm{~N}$, (b) $\mathrm{Fe}_{4} \mathrm{~N}$. 


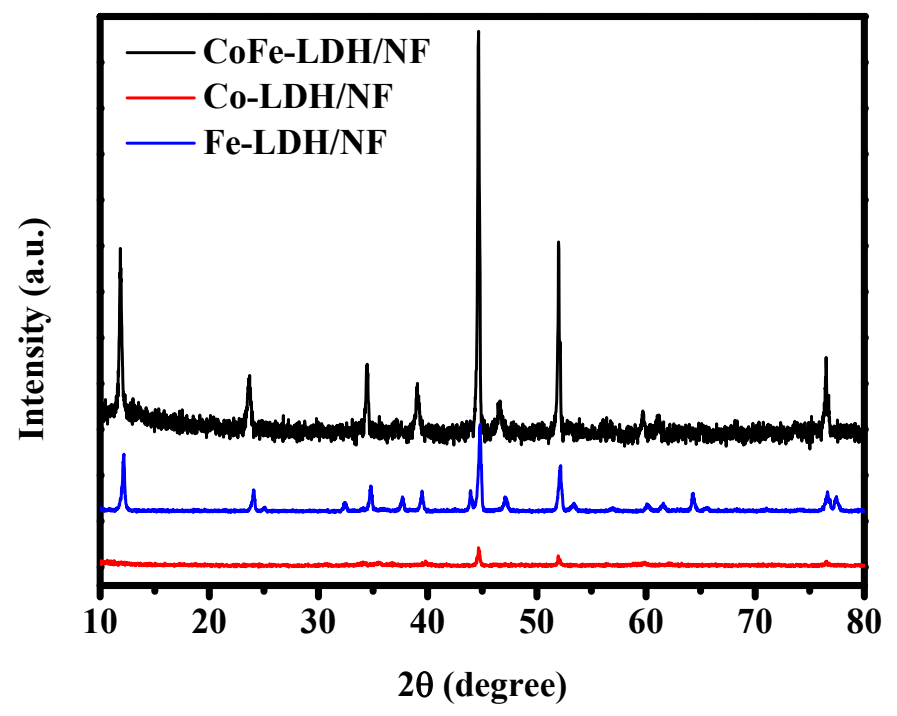

Figure S6. XRD patterns of the CoFe-LDH/NF, Co-LDH/NF, and Fe-LDH/NF. 


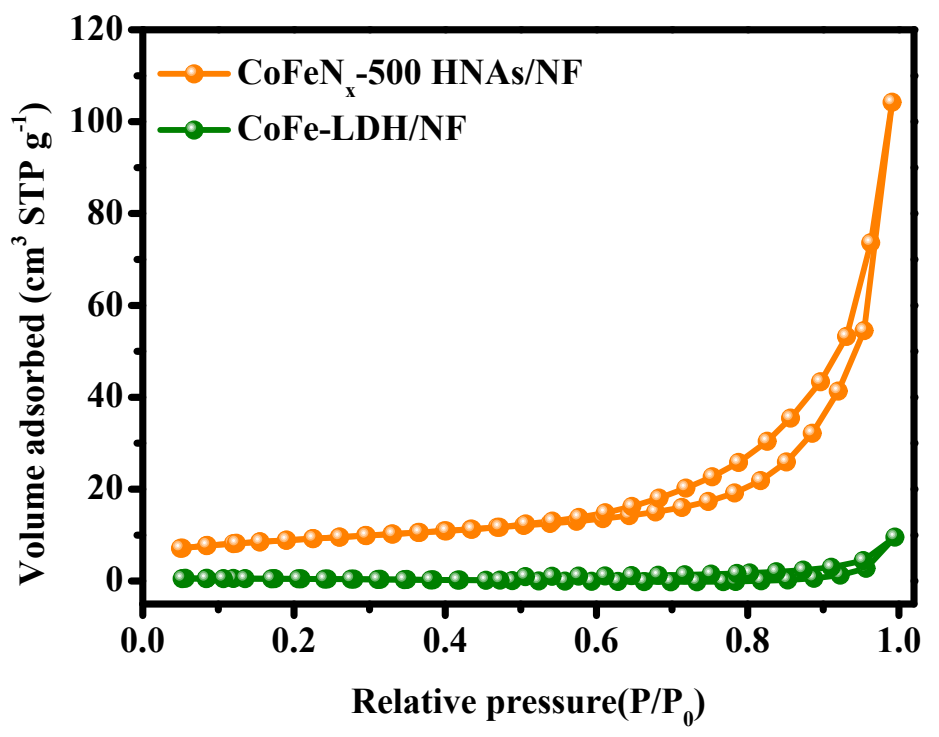

Figure S7. Nitrogen sorption isotherm of $\mathrm{CoFe}-\mathrm{LDH} / \mathrm{NF}$ and $\mathrm{CoFeN}_{x}-500 \mathrm{HNAs} / \mathrm{NF}$ samples. 

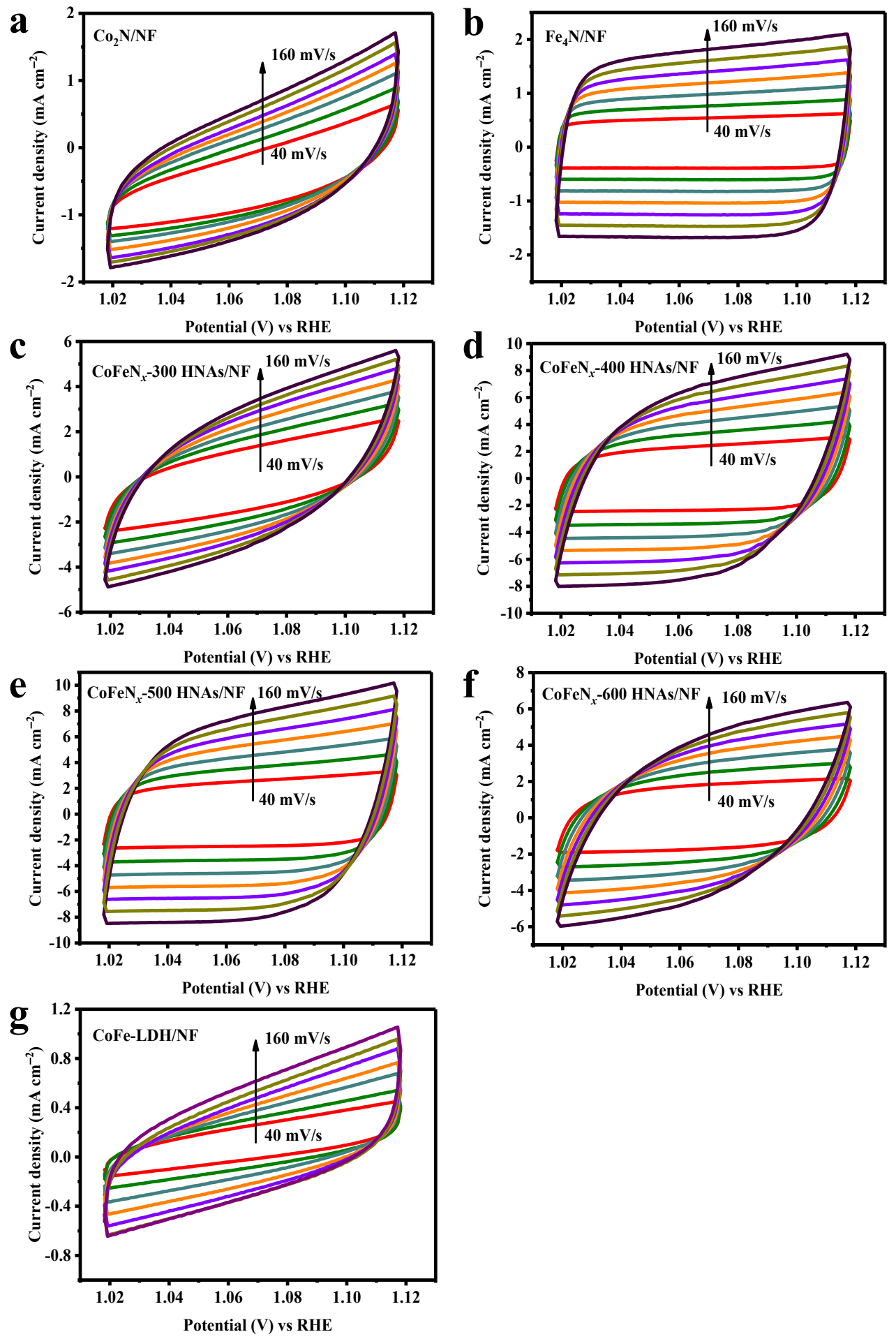

Figure S8. CV curves for OER: (a) $\mathrm{Co}_{2} \mathrm{~N} / \mathrm{NF}$, (b) $\mathrm{Fe}_{4} \mathrm{~N} / \mathrm{NF}$, (c) $\mathrm{CoFeN}_{x}-300 \mathrm{HNAs} / \mathrm{NF}$, (d) $\mathrm{CoFeN}_{x}-400 \mathrm{HNAs} / \mathrm{NF}$, (e) $\mathrm{CoFeN}_{x}-500 \mathrm{HNAs} / \mathrm{NF}$ and (f) $\mathrm{CoFeN}_{x}-600 \mathrm{HNAs} / \mathrm{NF}$ at different scan rates: $40,60,80,100,120,140$, and $160 \mathrm{mV} \mathrm{s}^{-1}$ from inside to outside. 


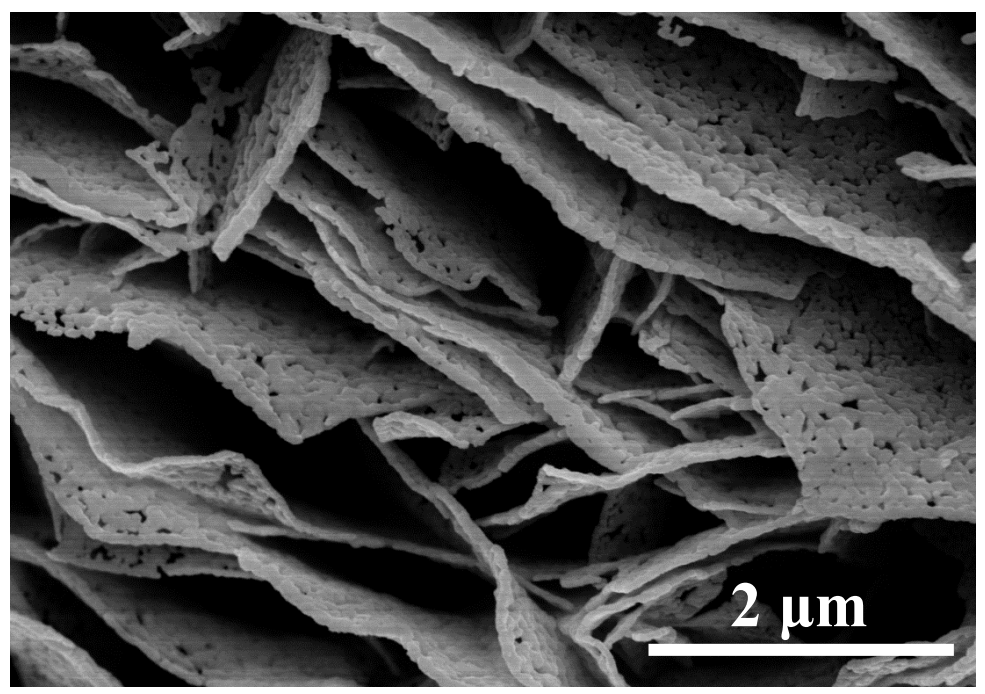

Figure S9. SEM image of $\mathrm{CoFeN}_{x}-500 \mathrm{HNAs} / \mathrm{NF}$ after long-time test. 

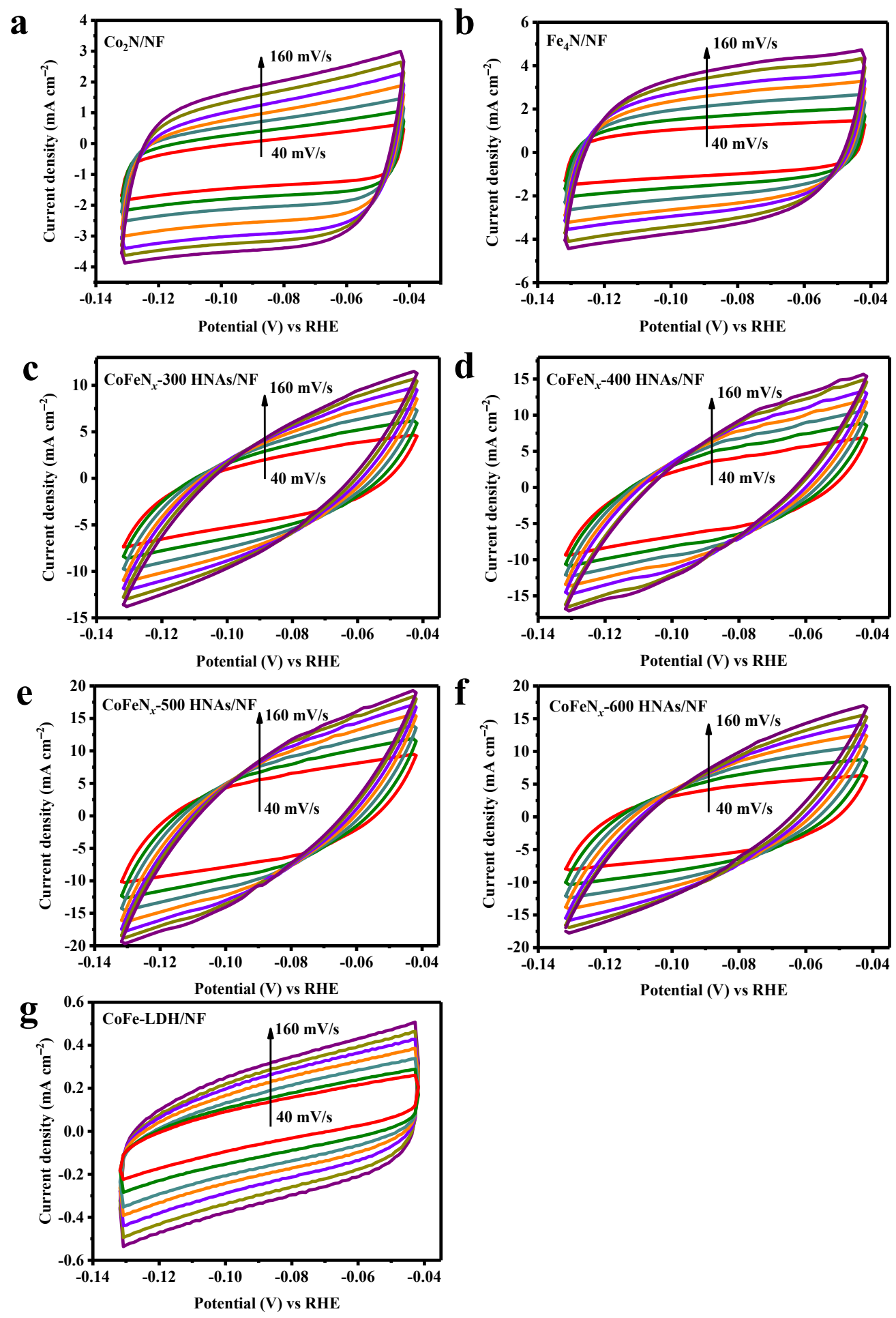

Figure S10. CV curves for HER: (a) $\mathrm{Co}_{2} \mathrm{~N} / \mathrm{NF}$, (b) $\mathrm{Fe}_{4} \mathrm{~N} / \mathrm{NF}$, (c) $\mathrm{CoFeN}_{x}-300 \mathrm{HNAs} / \mathrm{NF}$, (d) $\mathrm{CoFeN}_{x}-400 \mathrm{HNAs} / \mathrm{NF}$, (e) $\mathrm{CoFeN}_{x}-500 \mathrm{HNAs} / \mathrm{NF}$, (f) $\mathrm{CoFeN}_{x}-600 \mathrm{HNAs} / \mathrm{NF}$ and (g) $\mathrm{CoFe}-\mathrm{LDH} / \mathrm{NF}$ at different scan rates: 40, 60, 80, 100, 120, 140, and $160 \mathrm{mV} \mathrm{s}^{-1}$ from inside to outside. 


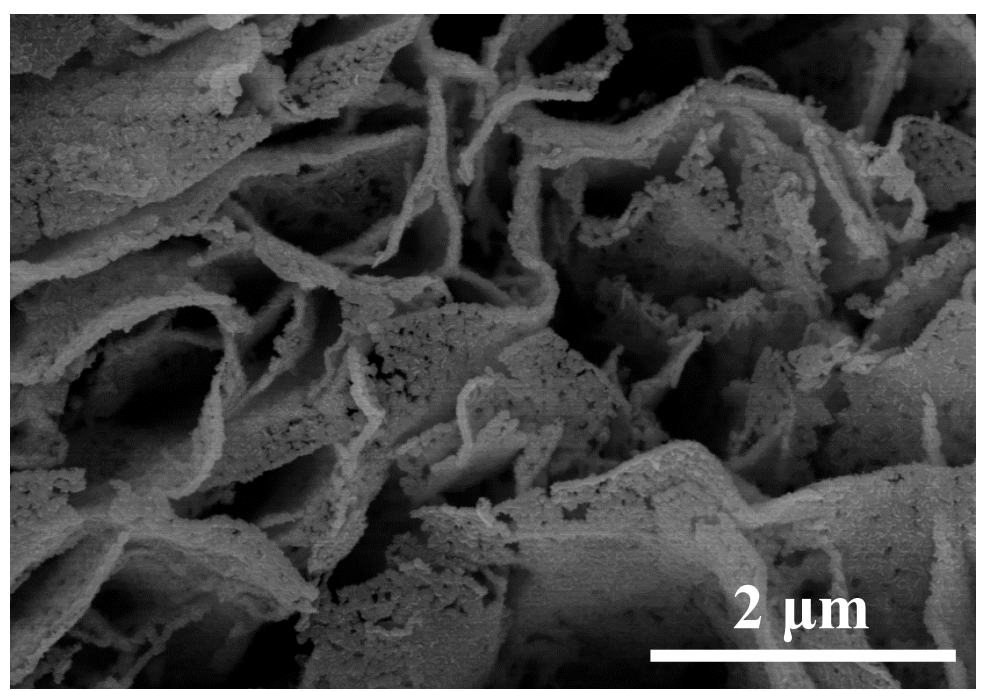

Figure S11. SEM images of $\mathrm{CoFeN}_{x}-500 \mathrm{HNAs} / \mathrm{NF}$ after long-time test. 


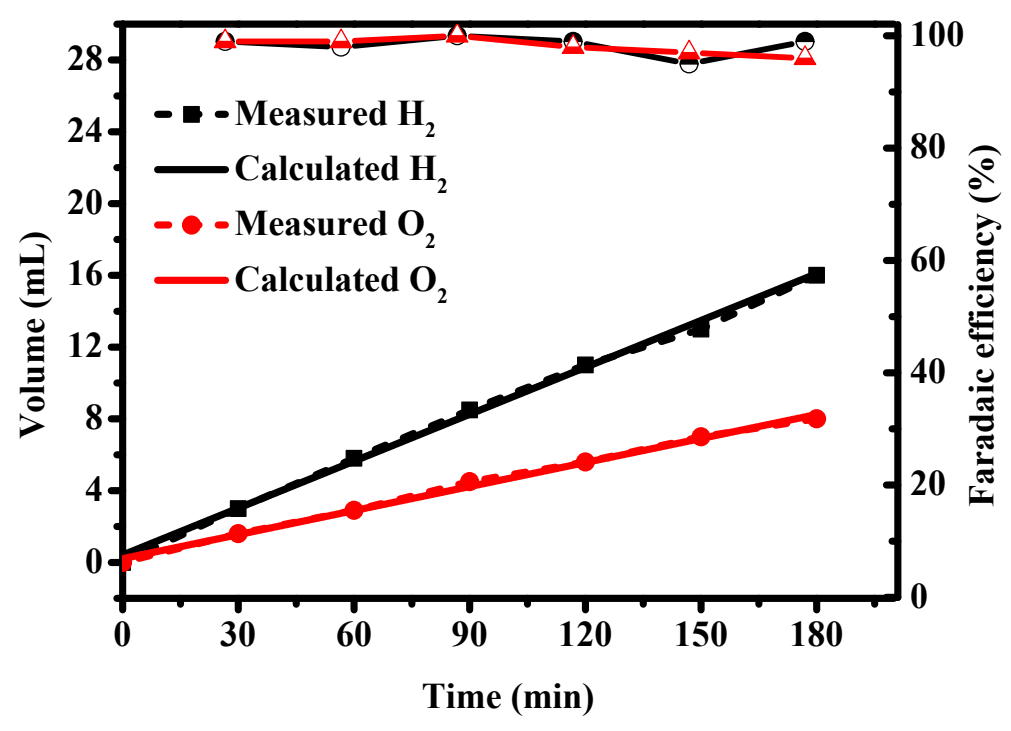

Figure S12. The volume of gas experimentally measured versus time on $\mathrm{CoFeN}_{x}-500$ HNAs/NF. Experimental and theoretical volumes of $\mathrm{H}_{2}$ and $\mathrm{O}_{2}$ by the $\mathrm{CoFeN}_{x}-500 \mathrm{HNAs} / \mathrm{NF}$ electrode, and the corresponding calculated Faradaic efficiency.

For the overall water splitting, the $\mathrm{CoFeN}_{x}-500 \mathrm{HNAs} / \mathrm{NF}$ was directly used as both cathodic and anodic electrodes for $\mathrm{H}_{2}$ and $\mathrm{O}_{2}$ production. And the Faradaic efficiency was calculated by evaluating the volume of experimentally quantified gas (water-gas displacing method at the current density of $10 \mathrm{~mA} \mathrm{~cm}-2$ ) with theoretically calculated gas:

$\eta_{\text {Faradaic efficiency }}=\left(\mathrm{V}_{\text {experimental }} / \mathrm{V}_{\text {theoretical }}\right) \times 100 \%(1)$

The theoretical volume of evolving gases can be calculated using the equation:

$\mathrm{V}_{\text {theoretical }}=\left(\mathrm{I} \times \mathrm{t} \times \mathrm{V}_{\mathrm{m}}\right) /(\mathrm{n} \times \mathrm{F})(2)$

where $\mathrm{I}$ is the current measured in the experiment, $\mathrm{t}$ is the recorded time, $\mathrm{V}_{\mathrm{m}}$ is the molar volume of $\mathrm{H}_{2}$ or $\mathrm{O}_{2}$ in $1 \mathrm{~mol} \mathrm{~L}^{-1}, \mathrm{n}$ is the number of electrons needed for $1 \mathrm{~mol}$ of $\mathrm{H}_{2}$ or $\mathrm{O}_{2}$ and $\mathrm{F}$ is the Faradaic constant $\left(96485 \mathrm{C} \mathrm{mol}^{-1}\right)$ 
Table S1. Elemental composition (atomic and weight percentage) obtained from EDS analysis.

\begin{tabular}{lccc}
\hline & $\mathrm{Co}$ & $\mathrm{Fe}$ & $\mathrm{N}$ \\
\hline Atomic \% & 20.64 & 24.08 & 10.01 \\
\hline Weight \% & 35.05 & 38.75 & 4.04 \\
\hline
\end{tabular}

Table S2. Elemental composition (atomic percentage) obtained from XPS analysis.

\begin{tabular}{cccc}
\hline & Co & Fe & N \\
\hline Atomic \% & 13.3 & 12.64 & 5.99 \\
\hline
\end{tabular}

Table S3. Elemental composition (atomic and weight percentage) obtained from ICP analysis.

\begin{tabular}{cccc}
\hline & Co & Fe & N \\
\hline Weight \% & 29.78 & 29.74 & 9.56 \\
\hline
\end{tabular}


Table S4. Comparisons of various state-of-the-art electrocatalysts for OER.

\begin{tabular}{|c|c|c|c|c|c|}
\hline Electrocatalysts & Substrate & $\begin{array}{c}\text { Electrolyte } \\
\text { (KOH) }\end{array}$ & $\begin{array}{c}\text { Overpotential } \\
\text { (mV vs. RHE) } \\
\text { a } 10,20 \text { or } 40 \\
\text { mA cm } \\
\end{array}$ & $\begin{array}{c}\text { Tafel } \\
\text { slope } \\
\text { mV/dec }\end{array}$ & Reference \\
\hline $\mathrm{CoFeN}_{x}$ HNAs & Nickel foam & $1.0 \mathrm{M}$ & $259(50)$ & 57.6 & This work \\
\hline $\mathrm{Co}_{3} \mathrm{~N}$ plate & Cobalt plate & $1.0 \mathrm{M}$ & $330(10)$ & 70 & $\begin{array}{l}\text { ACS Appl. } \\
\text { Mater. } \\
\text { Interfaces, } \\
\text { 2018, 10, } \\
\text { 22102-22109 }\end{array}$ \\
\hline CoN-1min & Nickel foam & $1.0 \mathrm{M}$ & $290(10)$ & 70 & $\begin{array}{l}\text { Angew. Chem. } \\
\text { Int. Ed., 2016, } \\
55,8670-8674\end{array}$ \\
\hline $\mathrm{Co}_{4} \mathrm{~N}$ & Carbon cloth & $1.0 \mathrm{M}$ & $257(10)$ & 44 & $\begin{array}{c}\text { Angew. Chem. } \\
\text { Int. Ed., 2015, } \\
54,14710- \\
14714\end{array}$ \\
\hline $\mathrm{Fe}_{x} \mathrm{~N}$ & Nickel foam & $1.0 \mathrm{M}$ & $257(20)$ & 44.5 & $\begin{array}{l}\text { ACS Catal., } \\
2017,7, \\
2052-2057\end{array}$ \\
\hline $\mathrm{Co}_{2} \mathrm{P}$ & $\begin{array}{c}\text { Carbon } \\
\text { nanotube }\end{array}$ & $1.0 \mathrm{M}$ & $280(10)$ & 72 & $\begin{array}{c}\text { J. Mater. Chem. } \\
\text { A, 2016, 4, } \\
15501-15510\end{array}$ \\
\hline $\mathrm{Co}_{3} \mathrm{Fe}_{1.5}-\mathrm{LDH}$ & Nickel foam & $1.0 \mathrm{M}$ & $286(10)$ & 45 & $\begin{array}{c}\text { Nanoscale, } \\
2017,9,16467- \\
16475\end{array}$ \\
\hline$(\mathrm{CoNi}-\mathrm{LDH} / \mathrm{Fe}-\mathrm{PP})_{30}$ & $\begin{array}{c}\text { Indium-tin-oxi } \\
\text { de }\end{array}$ & $1.0 \mathrm{M}$ & $264(10)$ & 37.6 & $\begin{array}{c}\text { J. Mater. Chem. } \\
\text { A, 2016, 4, } \\
11516-11523\end{array}$ \\
\hline $\mathrm{NiCo} / \mathrm{NiCoO}_{x} @ \mathrm{FeOOH}$ & Nickel foam & $1.0 \mathrm{M}$ & $278(10)$ & 47.5 & $\begin{array}{c}\text { Electrochimica } \\
\text { Acta, 2017, } \\
\text { 257, 1-8 }\end{array}$ \\
\hline $\mathrm{Ni}_{1.5} \mathrm{Fe}_{0.5} \mathrm{P}$ & 1 & $1.0 \mathrm{M}$ & $264(10)$ & 55 & $\begin{array}{c}\text { Nano Energy, } \\
2017,34,472- \\
480\end{array}$ \\
\hline $\mathrm{Ni}_{3} \mathrm{FeN} / \mathrm{r}-\mathrm{GO}-20$ & 1 & $1.0 \mathrm{M}$ & $270(10)$ & 54 & $\begin{array}{c}\text { ACS Nano, } \\
2018,12, \\
245-253\end{array}$ \\
\hline $\mathrm{S}-\mathrm{NiFe}_{2} \mathrm{O}_{4}$ & Nickel foam & $1.0 \mathrm{M}$ & $267(10)$ & 36.7 & $\begin{array}{c}\text { Nano Energy, } \\
2017,40,264- \\
273\end{array}$ \\
\hline
\end{tabular}




\begin{tabular}{|c|c|c|c|c|c|}
\hline $\mathrm{FeCo}-\mathrm{Co}_{4} \mathrm{~N} / \mathrm{N}-\mathrm{C}$ & / & $1.0 \mathrm{M}$ & $280(10)$ & 40 & $\begin{array}{c}\text { Adv. Mater., } \\
\text { 2017, 29, } \\
1704091\end{array}$ \\
\hline $\mathrm{Ni}_{3} \mathrm{FeN}$ & / & $1.0 \mathrm{M}$ & $280(10)$ & 46 & $\begin{array}{c}\text { Adv. Energy } \\
\text { Mater., 2016, 6, } \\
1502585\end{array}$ \\
\hline $\mathrm{Co}-\mathrm{Ni}-\mathrm{B}$ & Nickel foam & $1.0 \mathrm{M}$ & $313(10)$ & 120 & $\begin{array}{c}\text { J. Mater. Chem. } \\
\text { A, 2017, 5, } \\
\text { 12379-12384 }\end{array}$ \\
\hline $\mathrm{Ni}_{1.5} \mathrm{Fe}_{0.5} \mathrm{P}$ & Carbon fiber & $1.0 \mathrm{M}$ & $264(10)$ & 55 & $\begin{array}{c}\text { Nano Energy, } \\
2017,34,472- \\
480\end{array}$ \\
\hline $\mathrm{Co}-\mathrm{Ni}_{3} \mathrm{~N}$ & Carbon cloth & $1.0 \mathrm{M}$ & $307(10)$ & 57 & $\begin{array}{c}\text { Adv. Mater., } \\
2018,30 \\
1705516\end{array}$ \\
\hline $\mathrm{NiCo}_{2} \mathrm{~N}$ & Ni foam & $1.0 \mathrm{M}$ & $290(10)$ & 65 & $\begin{array}{c}\text { ChemSusChem, } \\
2017,10,4170- \\
4177\end{array}$ \\
\hline $\begin{array}{c}\mathrm{np}- \\
\left(\mathrm{Co}_{0.52} \mathrm{Fe}_{0.48}\right)_{2} \mathrm{P}\end{array}$ & I & $1.0 \mathrm{M}$ & $270(10)$ & 30 & $\begin{array}{c}\text { Energy } \\
\text { Environ. Sci., } \\
\text { 2016, 9, 2257- } \\
2261\end{array}$ \\
\hline $\mathrm{Ni}_{3} \mathrm{~N}-\mathrm{NiMoN}$ & Carbon cloth & $1.0 \mathrm{M}$ & $277(10)$ & 118 & $\begin{array}{c}\text { Nano Energy, } \\
2018,44,353- \\
363\end{array}$ \\
\hline
\end{tabular}


Table S5. The simulated series resistance $\left(R_{s}\right)$ and charge transfer resistance $\left(R_{c t}\right)$ based on the Nyquist plots (Figure 4e) in the presence of $\mathrm{CoFeN}_{x} \mathrm{HNAs} / \mathrm{NF}$ composites.

\begin{tabular}{cccccccc} 
& $\mathrm{Co}_{2} \mathrm{~N} / \mathrm{NF}$ & $\mathrm{Fe}_{4} \mathrm{~N} / \mathrm{NF}$ & $\mathrm{CoFe}-\mathrm{LDH} / \mathrm{NF}$ & $\mathrm{CoFeN}_{x}-300$ & $\mathrm{CoFeN}_{x}-400$ & $\mathrm{CoFeN}_{x}-500$ & $\mathrm{CoFeN}_{x}-600$ \\
& & & & $\mathrm{HNAs} / \mathrm{NF}$ & $\mathrm{HNAs} / \mathrm{NF}$ & $\mathrm{HNAs} / \mathrm{NF}$ & $\mathrm{HNAs} / \mathrm{NF}$ \\
\hline $\mathrm{R}_{\mathrm{s}}\left(\Omega \mathrm{cm}^{2}\right)$ & 1.84 & 2.12 & 1.29 & 2.1 & 1.13 & 0.834 & 1.29 \\
\hline $\mathrm{R}_{\mathrm{st}}\left(\Omega_{\mathrm{cm}}^{2}\right)$ & 1.81 & 1.49 & 1.27 & 1.07 & 0.83 & 0.726 & 1.06 \\
\hline
\end{tabular}


Table S6. Comparisons of various state-of-the-art electrocatalysts for HER.

\begin{tabular}{|c|c|c|c|c|c|}
\hline Electrocatalysts & Substrate & $\begin{array}{c}\text { Electrolyte } \\
\text { (KOH) }\end{array}$ & $\begin{array}{l}\text { Overpotential } \\
\text { (mV vs. RHE) } \\
@ 10 \text { mA cm }\end{array}$ & $\begin{array}{c}\text { Tafel } \\
\text { slope } \\
\text { mV/dec }\end{array}$ & Reference \\
\hline $\mathrm{CoFeN}_{x}$ HNAs & Ni foam & $1.0 \mathrm{M}$ & 200 & 60.64 & This work \\
\hline $\mathrm{Co}_{3} \mathrm{~N}$ plate & $\begin{array}{l}\text { Cobalt } \\
\text { plate }\end{array}$ & $1.0 \mathrm{M}$ & 230 & 101 & $\begin{array}{c}\text { ACS Appl. } \\
\text { Mater. } \\
\text { Interfaces, 2018, } \\
\text { 10, 22102-22109 }\end{array}$ \\
\hline $\mathrm{Cu}_{0.3} \mathrm{Co}_{2.7} \mathrm{P} / \mathrm{NC}$ & 1 & $1.0 \mathrm{M}$ & 220 & 122 & $\begin{array}{c}\text { Adv. Energy } \\
\text { Mater., 2017, 7, } \\
1601555\end{array}$ \\
\hline $\mathrm{Ni}_{1.5} \mathrm{Fe}_{0.5} \mathrm{P}$ & $\begin{array}{l}\text { Carbon } \\
\text { fiber }\end{array}$ & $1.0 \mathrm{M}$ & 282 & 125 & $\begin{array}{c}\text { Nano Energy, } \\
\text { 2017, 34, } \\
472-480\end{array}$ \\
\hline $\mathrm{Ni}_{2.5} \mathrm{Co}_{0.5} \mathrm{Fe}$ & 1 & $1.0 \mathrm{M}$ & 275 & 93 & $\begin{array}{c}\text { J. Mater. Chem. } \\
\text { A, 2016, 4, } \\
\text { 7245-7250 }\end{array}$ \\
\hline $\mathrm{Ni}_{3} \mathrm{~S}_{2}$ & Ni foam & $1.0 \mathrm{M}$ & 223 & l & $\begin{array}{c}\text { J. Am. Chem. } \\
\text { Soc., 2015, 137, } \\
\text { 14023-14026 }\end{array}$ \\
\hline $\mathrm{Co}-\mathrm{Ni}-\mathrm{B}$ & Ni foam & $1.0 \mathrm{M}$ & 205 & 1 & $\begin{array}{c}\text { J. Mater. Chem. } \\
\text { A, 2017, 5, } \\
\text { 12379-12384 }\end{array}$ \\
\hline $\mathrm{Co}-\mathrm{N}_{x} \mid \mathrm{P}-\mathrm{GC}$ & PEG & $1.0 \mathrm{M}$ & 260 & 115 & $\begin{array}{c}\text { Adv. Mater., } \\
2017,29, \\
1604480\end{array}$ \\
\hline FePNAs & $\begin{array}{l}\text { Carbon } \\
\text { cloth }\end{array}$ & $1.0 \mathrm{M}$ & 218 & 146 & $\begin{array}{l}\text { ACS Catal., } \\
2014,4,4065\end{array}$ \\
\hline $\mathrm{NiFe} \mathrm{LDH}$ & Ni foam & $1.0 \mathrm{M}$ & 210 & I & $\begin{array}{c}\text { Science, } 2014, \\
345,1593-1596\end{array}$ \\
\hline $\mathrm{NiCo}_{2} \mathrm{~S}_{4}$ & Ni foam & $1.0 \mathrm{M}$ & 210 & 59 & $\begin{array}{c}\text { Adv. Funct. } \\
\text { Mater., 2016, 26, } \\
\text { 4661-4672 }\end{array}$ \\
\hline $\mathrm{CoSe} / \mathrm{NiFeLDHs} / \mathrm{EG}$ & l & $1.0 \mathrm{M}$ & 260 & 160 & $\begin{array}{c}\text { Energy \& } \\
\text { Environ. } \\
\text { Sci., 2016, 9, } \\
478-483\end{array}$ \\
\hline $\mathrm{a}-\mathrm{CoSe} / \mathrm{Ti}$ & Ti mesh & $1.0 \mathrm{M}$ & 121 & 84 & $\begin{array}{c}\text { Chem. } \\
\text { Commun., 2015, } \\
\text { 51, 16683-16686 }\end{array}$ \\
\hline
\end{tabular}


Table S7. The exchange current density of $\mathrm{CoFeN}_{x}$ HNAs/NF composites.

\begin{tabular}{cccccccc}
\hline & $\mathrm{Co}_{2} \mathrm{~N} / \mathrm{NF}$ & $\mathrm{Fe}_{4} \mathrm{~N} / \mathrm{NF}$ & $\mathrm{CoFeN}_{x}-300$ & $\mathrm{CoFeN}_{x}-400$ & $\mathrm{CoFeN}_{x}-500$ & $\mathrm{CoFeN}_{x}-600$ & $\mathrm{CoFe}-\mathrm{LD}$ \\
\hline $\mathrm{j}_{\mathrm{o}}$ & & & $\mathrm{HNAs} / \mathrm{NF}$ & $\mathrm{HNAs} / \mathrm{NF}$ & $\mathrm{HNAs} / \mathrm{NF}$ & $\mathrm{HNAs} / \mathrm{NF}$ & $\mathrm{H} / \mathrm{NF}$ \\
\hline
\end{tabular}


Table S8. Comparison of TOF values about $\mathrm{CoFeN}_{x}$ HNAs/NF composites catalysts. TOFs are estimated by assuming that all the metal ions are active for catalysis, and the current density is at the overpotential of $200 \mathrm{mV}$ for $\mathrm{CoFeN}_{x} \mathrm{HNAs} / \mathrm{NF}$ composites, following the equation of TOF $=(j \times A) /(4 \times F \times m)$ for OER and TOF $=(j \times A) /(2 \times F \times m)$ for HER.

\begin{tabular}{cccccccc}
\hline TOF & $\mathrm{Co}_{2} \mathrm{~N} / \mathrm{NF}$ & $\mathrm{Fe}_{4} \mathrm{~N} / \mathrm{NF}$ & $\mathrm{CoFe}-\mathrm{LDH}$ & $\mathrm{CoFeN}_{x}-300$ & $\mathrm{CoFeN}_{x}-400$ & $\mathrm{CoFeN}_{x}-500$ & $\mathrm{CoFeN}_{x}-600$ \\
\hline OER $\left(10^{-3} \mathrm{~s}^{-1}\right)$ & 4.64 & 5.896 & 0.723 & 8.949 & 12.7 & 23.5 & 9.957 \\
\hline HER $\left(10^{-3} \mathrm{~s}^{-1}\right)$ & 2.711 & 2.127 & 0.968 & 2.9 & 3.967 & 6.358 & 3.451 \\
\hline
\end{tabular}


Table S9. The simulated series resistance $\left(R_{s}\right)$ and charge transfer resistance $\left(R_{c t}\right)$ based on the Nyquist plots (Figure 5e) in the presence of $\mathrm{CoFeN}_{x} \mathrm{HNAs} / \mathrm{NF}$ composites.

\begin{tabular}{ccccccccc}
\hline & $\mathrm{Co}_{2} \mathrm{~N} / \mathrm{NF}$ & $\mathrm{Fe}_{4} \mathrm{~N} / \mathrm{NF}$ & $\mathrm{CoFe}-\mathrm{LDH} / \mathrm{NF}$ & $\mathrm{CoFeN}_{x}-300$ & $\mathrm{CoFeN}_{x}-400$ & $\mathrm{CoFeN}_{x}-500$ & $\mathrm{CoFeN}_{x}-600$ \\
& & & & $\mathrm{HNAs} / \mathrm{NF}$ & $\mathrm{HNAs} / \mathrm{NF}$ & $\mathrm{HNAs} / \mathrm{NF}$ & $\mathrm{HNAs} / \mathrm{NF}$ \\
\hline $\mathrm{R}_{\mathrm{s}}\left(\Omega \mathrm{cm}^{2}\right)$ & 2.28 & 2.1935 & 2.095 & 1.79 & 2.01 & 1.71 & 2.11 \\
\hline $\mathrm{R}_{\mathrm{st}}\left(\Omega \mathrm{cm}^{2}\right)$ & 2.05 & 3.25 & 5.068 & 1.85 & 1.56 & 0.91 & 1.44 \\
\hline
\end{tabular}

